\title{
Prompt-Gamma Monitoring of Proton- and Carbon-Therapy. Combined Development of Time-of-Flight Collimated- and Compton-Cameras
}

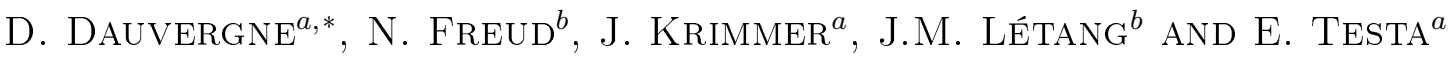 \\ ${ }^{a}$ Institut de Physique Nucléaire de Lyon, Université de Lyon, Université Lyon 1, CNRS/IN2P3, UMR5822, \\ F-69622 Villeurbanne, France \\ ${ }^{b}$ CREATIS, Université de Lyon, Université Lyon 1, CNRS UMR5220, INSERM U1044, INSA-Lyon, \\ Centre Léon Bérard, F-69622 Villeurbanne, France
}

\begin{abstract}
Prompt-gamma imaging during ion therapy has proven its ability to control the ion range in real time. The achievable precision is of the order of the millimeter for a single spot in proton pencil beam scanning. Collimated gamma cameras have been developed, that are close to clinical application. The Compton cameras are also under development in various laboratories. Time of flight enables the reduction of the background due to other prompt radiations.
\end{abstract}

DOI: 10.12693 /APhysPolA.127.1445

PACS: 29.40.Mc, 07.85.Fv, 87.53.Bn, 87.56.Fc, 87.56.- $\mathrm{v}$

\section{Introduction}

The aim of cancer radiotherapy is to deposit a maximum of dose in the targeted tumor, while minimizing the dose to surrounding healthy tissues, especially to the organs at risk. The therapeutic window has to be set in conditions where local control probability is at maximum, whereas the probability of complications - such as radio-induced secondary cancers - is at minimum. Although high contrast between dose inside and outside a tumor can be reached in modern X-ray therapy, this will necessarily be done at the expense of a large volume of irradiated tissue, since only a small fraction of the energy is actually deposited in the tumor volume for deep-seated tumors. In contrast, ions have a finite range in matter and deposit a maximum of energy at the end of their range. Thus the corresponding advantages of ion-therapy are (i) a better targeting of the tumor volume, with enhanced ratio of energy deposited inside the tumor to the energy delivered to healthy tissues, (ii) a unique lateral and longitudinal ballistic precision enabling a sparing of organs at risk and (iii) in the case of light ions like carbon, enhanced radiobiological effectiveness relative to photons or protons in the tumor volume makes carbon therapy suitable for radioresistant tumors.

Actually, the ballistic precision of particle-therapy makes this technique quite sensitive to any cause of deviation with respect to treatment planning (due to mispositioning, organ motion or anatomic evolution between fractions), or uncertainties in the planning itself (due to relative stopping power estimation from CT scans). Nowadays, safety margins are applied in order to ensure

${ }^{*}$ corresponding author; e-mail: d.dauvergne@ipnl.in2p3.fr that the target volume is effectively irradiated with the prescribed dose. In proton-therapy such margins are typically $\approx 2.5-3 \%+2-3 \mathrm{~mm}$ [1]. The reduction of margins could be obtained either by improving the precision of the treatment planning or by online control of the deposited dose. Proton radiography may provide direct measurement of the electron density during planning, and also control of the position before treatment [2]. However, this imaging modality is still at a research stage, and its implementation would require beams that traverse the patient, i.e. often with higher energies than currently available.

\section{Rationale}

Online control of the treatment, and in particular the measurement of the range, can be performed by means of secondary radiations issued from nuclear reactions. Other imaging modalities, like ion-induced ultrasounds, appear promising in some specific cases [3]. The main imaging modality that has already been applied clinically is positron emission tomography (PET) [4]. Positron emitters are created during fragmentation of projectiles (carbon therapy) or target nuclei (any kind of particle therapy). The observation of the beta decay is delayed, with respect to the irradiation, by the lifetime of the radioactive isotopes, which extends from a few seconds $\left({ }^{10} \mathrm{C}\right)$ to few minutes $\left({ }^{11} \mathrm{C},{ }^{15} \mathrm{O} \ldots\right)$. Offline PET imaging devices can be used right after a treatment session, and give access to the delayed emission of longlived isotopes. However, this represents an a posteriori verification of the irradiated volume, and the images suffer from relatively low statistics and from blurring due to metabolism in blood-irrigated organs. Online PET systems are able to acquire short-lived isotope decays during beam pauses and just after the treatment stops, which limits washout effects. However, this implies lim- 
ited solid-angle devices to be compliant with the irradiation and patient positioning systems. Furthermore, PET acquisitions will still require acquisition times longer than the irradiation to reach sufficient statistics, and thus PET will anyway remain an a posteriori control.

In contrast, prompt-gamma (PG) imaging presents some assets:

- PG emission is correlated to the primary ion range $[5,6]$;

- transmission of high energy photons through the patient without scattering is likely to occur [7];

- a sufficiently large number of photons are emitted to ensure millimetric control of proton range for a single pencil beam spot [8-10], and at an energy slice level for carbon ions, in the distal part of the irradiated volume;

- real-time information is obtainable with collimated devices, without sophisticated reconstruction;

- little dependence is expected on the tissue and the organs under treatment (unlike ultrasound or MRI for instance); therefore, PG imaging can be considered as generally applicable;

- PG imaging is applicable for proton and carbon beams, at the expense of time-of-flight selection for carbon therapy [11].

PG emission is synchronized with the beam delivery, as most of other secondary radiations. Therefore, their detection is somehow hindered by a background due to other particles, mainly neutrons. As neutrons are subject to scattering with high cross-sections, they do not keep the information on their creation vertex position. Discrimination techniques such as time of flight (TOF), provided the distance of observation to the beam is large enough, help in reducing this background. In the case of carbon ions, such discrimination is necessary due to the large yield of neutrons.

Using collimated devices and TOF discrimination, our collaboration collected a series of PG profiles at various proton and carbon beams, with water and poly(methyl methacrylate) (PMMA) targets. Consistent analysis, using the same residual background subtraction method, has led to typical $\mathrm{PG}$ yields above $1 \mathrm{MeV}$ of $\approx 3 \times$ $10^{-3} \mathrm{~cm}^{-1}$ per proton and $\approx 2 \times 10^{-2} \mathrm{~cm}^{-1}$ per carbon ion in a PMMA target [12]. These yields are those directly induced by the projectiles (i.e. measured at the target entrance). A built-up along the ion path is observed, that is due to the production by secondary particles, and partly to the increase of fragmentation cross-sections when the energy decreases.

Detailed spectral analysis of the discrete PG lines provides information on the chemical composition of the irradiated volume, in particular the relative weights of carbon and oxygen along the beam path [13]. Verburg et al. combined the spectral information with the spatial information on the emission point, using a collimated detection setup [14]. By means of multi-parametric analysis, they have shown that both chemical composition in the field of view of the slit and ion range can be measured. The latter work also showed that individ- ual gamma lines result from different energy-differential cross-sections, and therefore the shape of gamma emission profiles may vary upon which lines are considered. This may render more complex the prediction and interpretation of $\mathrm{PG}$ profiles in heterogeneous media.

In addition to $\mathrm{PG}$ profile measurements, it was reported recently that $\mathrm{PG}$ timing also gives access to the transit time of ions inside the patient, and thus to the ion range [15]. A non-collimated device measures a time profile with respect to the time of impact of the ions on the patient, which is related to the duration of the path. Sub-centimeter variation of the ion range is detectable as a few tens of picosecond variation of the time spectrum.

Actually, PG imaging of ion ranges remains quite challenging in real treatment conditions: the energy range above $1 \mathrm{MeV}$ makes it incompatible with current SPECT imaging devices. Moreover, the high instantaneous count rates during treatments require sophisticated detection and acquisition devices, able to operate in noisy neutronbackground. Last, online devices have to be compliant with patient positioning and beam delivery systems (e.g. gantries). This explains why PG imaging has not yet been implemented clinically, although the idea was raised more than ten years ago [16].

\section{Device developments}

Several groups around the world started to build or design PG imaging prototypes, based either on collimated devices or Compton cameras, in particular during ENVISION [17], a joined effort between European teams, including studies on PG, PET and secondary proton imaging. Collimated cameras are of two types: on the one hand, knife-edge single-slit cameras $[8,18]$ present the advantage of compactness, simple collimation and detection. On the other hand, multi-slit cameras enable non-restricted field of view and potentially better spatial resolution $[10,19]$.

The Compton cameras should be well adapted for the present application, since Compton scattering is by far the dominant interaction process of PGs. Compton imaging is based on the determination of the Compton scattering angle of an incident photon, for which either the energy is known (general case in medical imaging) or from a point-like polychromatic source (general case in gamma-astrophysics). The particular application in the control of hadrontherapy combines both disadvantages of an extended- and polychromatic source. Therefore, an ideal Compton camera would require the measurement of the full energy and position of the scattered photon in an absorber detector and of the electron in the scatter detector, or a second Compton scattering in a second detector, or the full tracking of the scattered electron (angle and energy). Several prototypes have been under study over the last years [20-24], combining the various options.

In order to realize a multi-collimated camera prototype, simulations and data acquired with single and multi-collimated cameras at proton and carbon beams were used to optimize the detection configuration. 
The retrieval precision of the $\mathrm{PG}$ profile fall-off, or of the prompt-gamma profile length (PGPL), characterizes the precision of a given setup with which these quantities are measured for a given statistics. Actually, it has been shown that statistics on the detected photons is the main parameter governing this precision in homogeneous target phantoms $[8,9]$. With heterogeneous targets, however, one has to consider a compromise between high statistics and spatial resolution, since heterogeneities located close to the patient entrance or the Bragg peak may lead to distorted profiles, and thus misinterpretations. Our technical solution consists in a multi-collimated camera, with use of TOF to maximize the contrast to background ratio. Simulations were undertaken to optimize the collimator geometry, by optimizing the fall-off retrieval precision [10]. The localization of the photons behind multi-collimation slits was measured by means of segmented detectors, which validated the use of streaked BGO blocks as detectors [22]. The detector under construction should allow for a determination of the PGPL with millimetric precision at the scale of a single proton spot in pencil beam scanning delivery $\left(\approx 10^{8}\right.$ incident protons) in the distal region of the tumor.

In parallel, a Compton camera has been developed. We adopted the configuration of a two-stage Compton camera, i.e. with only a single scattering stage. Indeed, simulations showed that the total efficiency of a single-scattering stage camera is more than one order of magnitude higher than a double-scattering one. In addition, an absorber configuration with highest possible effective atomic number $Z_{\text {eff }}$ makes the localization in the absorber detector quite precise [25]. The resolution calculated on a point spread function (PSF) was degraded from $6 \mathrm{~mm}$ to $8.3 \mathrm{~mm}$ FWHM when using single scattering [26] instead of double scattering [27]. The detector under assembly comprises a stack of 7 double sided silicon strip detectors (DSSD) as a scatter detector. Each DSSD is $96 \times 9.6 \times 2 \mathrm{~mm}^{3}$ in size, with 64 strips on each side. Dedicated low-noise, large dynamics and high count-rate capability micro-electronics front-end was designed and successfully tested [28]. The absorber detector is the same as for the collimated camera, i.e. it is made of an assembly of BGO blocks, each block being streaked in order to have a position reading with a pitch of $4 \mathrm{~mm}$ in $X$ and $Y$. The size of an individual block is $34 \times 34 \times 30 \mathrm{~mm}^{3}$, and its readout is made by means of 4 photomultiplier tubes. The front-end acquisition is made of analog sampling modules with $1 \mathrm{GHz}$ sampling digitizers. The global acquisition system is made with micro-TCA standard. A single AMC card communicates with $4.8 \mathrm{GHz}$ input/ouput rate capability with the 34 front-end modules.

A beam triggering system may be necessary for longpulsed beams to perform TOF measurements, and then reduce the background induced by neutrons and other particles. Therefore, we developed a beam hodoscope, consisting of two planes of scintillating fibers, read by photomultipliers. It comprises $2 \times 128$ square fibers of
$1 \mathrm{~mm}$ size. The light is transported by means of optical fibers toward 64-channel multi-anode PMTs. 8 PMTs are used to read the 256 fibers on each extremity. A 32channel ASIC readout front-end of the PMTs comprises a current-conveyor with comparator and analog output, and a delayed-locked-loop digital timing with 60 ps resolution [29].

\section{Conclusions}

Prompt-gamma imaging has proven its ability to monitor online hadrontherapy. Real-time verification of the range with millimetric accuracy is achievable during protontherapy at the scale of a single pencil beam, i.e. at the first millisecond of the treatment. Thanks to the developments undertaken during the European project ENVISION and by several other groups all over the world, PGI is now close to clinical translation, and will account for the beam time structure and intensities of the large variety of beam delivery modes. The clinical-sized collimated camera and Compton camera that are currently developed by the French collaboration aim at maximized performances in terms of field of view, spatial resolution and contrast-to-noise ratio using time-of-flight discrimination. The cameras will be tested under clinical conditions soon.

\section{Acknowledgments}

These research developments have been supported by the Regional Program for Research in Hadrontherapy (PRRH, under CPER 2007-13 funding), the European FP7 projects ENVISION (grant agreement no. 241851), ENTERVISION (grant agreement no. 264552), ULICE (grant agreement no. 228436) and the ANR Gamhadron project (ANR-09-BLAN-0106). This work is performed within the framework of the LABEX PRIMES (ANR-11LABX-0063) and France Hadron (ANR-11-INBS-0007).

\section{References}

[1] H. Paganetti, Phys. Med. Biol. 57, R99 (2012).

[2] H.F.-W. Sadrozinski, R.P. Johnson, S. Macafee, A. Plumb, D. Steinberg, A. Zatserklyaniy, V.A. Bashkirov, R.F. Hurley, R.W. Schulte, Nucl. Instrum. Methods Phys. Res. A 699, 205 (2013).

[3] W. Assmann, S. Kellnberger, S. Reinhardt, S. Lehrack, A. Edlich, P. G. Thirolf, M. Moser, G. Dollinger, M. Omar, V. Ntziachristos and K. Parodi, Med. Phys. 42, 567 (2015).

[4] W. Enghardt, P. Crespo, F. Fiedler, R. Hinz, K. Parodi, J. Pawelke, F. Pönisch, Nucl. Instrum. Methods Phys. Res. A 525, 284 (2004).

[5] C.-H. Min, C.H. Kim, M.-Y. Youn, J.-W. Kim, Appl. Phys. Lett. 89, 183517 (2006).

[6] E. Testa, M. Bajard, M. Chevallier, D. Dauvergne, F. Le Foulher, N. Freud, J.M. Létang, J.C. Poizat, C. Ray, M. Testa, Appl. Phys. Lett. 93, 093506 (2008).

[7] M. Moteabbed, S. Espana, H. Paganetti, Phys. Med. Biol. 56, 1063 (2011). 
[8] J. Smeets, F. Roellinghoff, D. Prieels, F. Stichelbaut, A. Benilov, P. Busca, C. Fiorini, R. Peloso, M. Basilavecchia, T. Frizzi, J.C. Dehaes, A. Dubus, Phys. Med. Biol. 57, 3371 (2012).

[9] F. Roellinghoff, A. Benilov, D. Dauvergne, G. Dedes, N. Freud, G. Janssens, J. Krimmer, J.M. Létang, M. Pinto, D. Prieels, C. Ray, J. Smeets, F. Stichelbaut, E. Testa, Phys. Med. Biol. 59, 1327 (2014).

[10] M. Pinto, D. Dauvergne, N. Freud, J. Krimmer, J.M. Létang, C. Ray, F. Roellinghoff, E. Testa, Phys. Med. Biol. 59, 7653 (2014).

[11] M. Testa, M. Bajard, M. Chevallier, D. Dauvergne, P. Henriquet, F. Le Foulher, C. Ray, E. Testa, N. Freud, J.M. Létang, M.-H. Richard, S. Karkar, R. Plescak, D. Schardt, Radiat. Environ. Biophys. 49,337 (2010).

[12] M. Pinto, M. Bajard, S. Brons, M. Chevallier, D. Dauvergne, G. Dedes, M. De Rydt, N. Freud, J. Krimmer, C. La Tessa, J.M. Létang, K. Parodi, R. Pleskac, D. Prieels, C. Ray, I. Rinaldi, F. Roellinghoff, D. Schardt, E. Testa, M. Testa, Phys. Med. Biol. 60, $565(2015$.

[13] J.C. Polf, R. Panthi, D.S. Mackin, M. McCleskey, A. Saastamoinen, B.T. Roeder, S. Beddar, Phys. Med. Biol. 58, 5821 (2013).

[14] J.M. Verburg, K. Riley, T. Bortfeld, J. Seco, Phys. Med. Biol. 58, L37 (2013).

[15] C. Golnik, F. Hueso-González, A. Müller, P. Dendooven, W. Enghardt, F. Fiedler, T. Kormoll, K. Roemer, J. Petzoldt, A. Wagner, G. Pausch, Phys. Med. Biol. 59, 5399 (2014).

[16] F. Stichelbaut, Y. Jongen, in: 39th Meeting of the Particle Therapy Co-Operative Group, San Francisco 2003, unpublished.

[17] http://envision.web.cern.ch/ENVISION/.

[18] V. Bom, L. Joulaeizadeh, F. Beekman, Phys. Med. Biol. 57, 297 (2012).

[19] C.H. Min, H.R. Lee, C.H. Kim, S.B. Lee, Med. Phys. 39, 2100 (2012).

[20] F. Hueso-González, C. Golnik, M. Berthel, A. Dreyer, W. Enghardt, F. Fiedler, K. Heidel, T. Kormoll, H. Rohling, S. Schöne, R. Schwengner, A. Wagner, G. Pausch, J. Instrum. 9, P05002 (2014).
[21] G. Llosá, J. Cabello, S. Callier, J. Gillam, C. Lacasta, M. Rafecas, L. Raux, C. Solaz, V. Stankova, C. de La Taille, M. Trovato, J. Barrio, Nucl. Instrum. Methods Phys. Res. A 718, 130 (2013).

[22] J. Krimmer, J.-L. Ley, C. Abellan, J.-P. Cachemiche, L. Caponetto, X. Chen, M. Dahoumane, D. Dauvergne, N. Freud, B. Joly, D. Lambert, L. Lestand, J.M. Létang, M. Magne, H. Mathez, V. Maxim, G. Montarou, C. Morel, M. Pinto, C. Ray, V. Reithinger, E. Testa, Y. Zoccarato, Nucl. Instrum. Methods Phys. Res. A, in press.

[23] C.H. Kim, J.H. Park, H. Seo, H.R. Lee, Med. Phys. 39, 6523 (2012).

[24] S. Kurosawa, H. Kubo, K. Ueno, S. Kabuki, S. Iwaki M. Takahashi, K. Taniue, N. Higashi, K. Miuchi, T. Tanimori, D. Kim, J. Kim, Curr. Appl. Phys. 12, 364 (2012).

[25] M.-H. Richard, M. Dahoumane, D. Dauvergne, G. Dedes, M. De Rydt, N. Freud, J.M. Letang, X. Lojacono, J. Krimmer, V. Maxim, G. Montarou, C. Ray, F. Roellinghoff, E. Testa, A.H. Walenta, IEEE Trans. Nucl. Sci. 59, 1850 (2012).

[26] F. Roellinghoff, M.-H. Richard, M. Chevallier, J. Constanzo, D. Dauvergne, N. Freud, P. Henriquet, F. Le Foulher, J.M. Létang, G. Montarou, C. Ray, E. Testa, M. Testa, A.H. Walenta, Nucl. Instrum. Methods Phys. Res. A 648, S20 (2011).

[27] M.-H. Richard, M. Chevallier, D. Dauvergne, N. Freud, P. Henriquet, F. Le Foulher, J.M. Létang, G. Montarou, C. Ray, F. Roellinghoff, E. Testa, M. Testa, A.H. Walenta, IEEE Trans. Nucl. Sci. 58, 87 (2010)

[28] M. Dahoumane, D. Dauvergne, J. Krimmer, H. Mathez, C. Ray, E. Testa, A.H. Walenta, Y. Zoccarato, in: Nuclear Science Symp. and Medical Imaging Conf. (NSS/MIC), IEEE, Annaheim (2012), p. 1445.

[29] S. Deng, D. Dauvergne, G.-N. Lu, H. Mathez, Y. Zoccarato, J. Instrum. 8, C01047 (2013). 